\title{
Transcriptomic and epigenomics atlas of myotubes reveals insight into the circadian control of metabolism and development
}

\author{
Ali Altıntaşs, 1(i), Rhianna C Laker ${ }^{\ddagger 1}{ }^{\ddagger}$, Christian Garde1, Romain Barrès ${ }^{1}$ (D) \& Juleen R \\ Zierath*,1,2 (iD) \\ ${ }^{1}$ Section for Integrative Physiology, The Novo Nordisk Foundation Center for Basic Metabolic Research, University of Copenhagen, \\ Copenhagen, Denmark \\ ${ }^{2}$ Section for Integrative Physiology, Department of Molecular Medicine \& Surgery, Karolinska Institutet, Stockholm, Sweden \\ *Author for correspondence: Juleen.Zierath@ki.se \\ $¥$ Authors contributed equally
}

\begin{abstract}
Aim: Innate circadian rhythms are critical for optimal tissue-specific functions, including skeletal muscle, a major insulin-sensitive tissue responsible for glucose homeostasis. We determined whether transcriptional oscillations are associated with CpG methylation changes in skeletal muscle. Materials \& methods: We performed rhythmicity analysis on the transcriptome and $\mathrm{CpG}$ methylome of circadian synchronized myotubes. Results: We identified several transcripts and $\mathrm{CpG}$-sites displaying oscillatory behavior, which were enriched with Gene Ontology terms related to metabolism and development. Oscillating CpG methylation was associated with rhythmic expression of 31 transcripts. Conclusion: Although circadian oscillations may be regulated by rhythmic DNA methylation, strong rhythmic associations between transcriptome and CpG methylation were not identified. This resource constitutes a transcriptomic/epigenomic atlas of skeletal muscle and regulation of circadian rhythms.
\end{abstract}

First draft submitted: 17 December 2019; Accepted for publication: 5 February 2020; Published online: 11 March 2020

Keywords: $\mathrm{C} 2 \mathrm{C} 12 \bullet$ circadian $\bullet$ epigenetics $\bullet$ methylation $\bullet$ muscle $\bullet$ myotube $\bullet$ transcriptomics

Circadian rhythms allow organisms to optimize various behavioral and physiological functions throughout the $24 \mathrm{~h}$ day-night cycle. Peripheral tissues are under the control of both the central clock, entrained by light signaling through the optical nerve to the suprachiasmatic nucleus and an intrinsic clock, entrained by a variety of environmental and systemic cues, including dietary factors, physical activity, metabolic demand and circulating hormones [1,2]. Mounting evidence indicates that when human behavior becomes out-of-sync with the central and peripheral clocks (e.g., after shift work or jet lag), whole body metabolism is perturbed and the risk for the development of Type 2 diabetes is increased [3-5]. Understanding the genomic mechanisms by which Zeitgebers (time givers) regulate the circadian expression of genes in metabolic tissues may give insight into disease mechanism for insulin resistance and altered metabolic homeostasis in type 2 diabetes.

The intrinsic mammalian circadian clock is comprised of the transcription factors ARNTL (also known as BMAL1) and CLOCK [6]. These molecules dimerize and bind the E-box motif in the promoter of Nr1d1 (also known as Rev-Erb $\alpha$ ) to drive Nr1d1 transcription. In turn, NR1D1 feeds back and binds the DNA response element in the Arntl promoter and blocks transcription. The binding of NR1D1 is balanced by competitive binding of ROR $\alpha$, which has an opposite function to drive the expression of ARNTL. Concomitantly, ARNTL and CLOCK drive the expression of the Period (Per1, Per2 and Per3) and Cryptochrome (Cry1 and Cry2) genes, whose protein products translocate to the nucleus to block Arntl activity. These molecular cycles oscillate with a $24 \mathrm{~h}$ period that correspond with the day-night cycle and function as a transcription-translation feedback loop. ARNTL and CLOCK drive the expression of many genes that function in circadian regulation, but also bind to E-box motifs to regulate transcription of genes that are not classically circadian. 
Skeletal muscle is responsible for $70-80 \%$ of insulin-stimulated glucose uptake, which is severely reduced with metabolic diseases such as Type 2 diabetes [7]. Mammalian skeletal muscle has an intrinsic molecular clock [8-10] and displays rhythmic expression of metabolic genes, such as $P d k 4, U c p 3$ and $P g c-1 \beta$, as well as genes involved in myogenesis, such as Myod1, Atrogin1 and Murf1 [11,12]. Thus, cellular growth and metabolism are partly under the control of the molecular clock machinery. On a functional level, isolated human muscle fibers [13] and mouse myotubes [14] demonstrate rhythmic oxygen consumption, concomitant with clock-dependent myokine secretion as reported for primary human myotubes [15]. Skeletal muscle loss of Arntl impairs whole body glucose homeostasis and insulin sensitivity and reduces the abundance of GLUT4 and various metabolic enzymes [16]. Thus, circadian rhythmicity is critical for metabolism and contractile function of skeletal muscle.

The oscillatory nature of the molecular clock is highly dependent on gene transcription and, consequently, epigenetic regulation has been implicated in the fine tuning of this process. Oscillating histone modifications have been described in circadian regulation of the liver metabolism [17-21]. However, the role of DNA methylation of cytosine bases in the transcriptional regulation of the mammalian circadian clock is unclear. Circadian disruption by shortening the length of day from 24 to $22 \mathrm{~h}$ alters promoter DNA methylation in the suprachiasmatic nucleus at 1294 regions and many thereby impact downstream processes, including mood, learning and memory [22]. Thus, changes in DNA methylation in response to circadian disruption may impact the central control of behavior. At the cellular level in liver, while circadian oscillations in histone modifications in promoters, gene bodies and enhancers have been reported [21], whole-genome bisulfite sequencing at two distinct circadian timepoints revealed promoter DNA methylation levels were relatively stable [21]. Nevertheless, a comprehensive analysis of genome-wide DNA methylation and transcriptional oscillations at multiple time points and in other peripheral tissues has not been reported.

The purpose of our study was to delineate the potential link between the circadian CpG methylation and the regulation of circadian gene expression in skeletal muscle. We performed a time-course analysis of the circadian response of gene expression in cultured skeletal muscle and determined parallel epigenetic regulation by assessing changes in DNA methylation at specific promoter regions that correspond to the circadian gene expression profiles. We examined genome-wide methylation across a time course to determine whether transcriptional oscillations are associated with and potentially regulated by rhythmic DNA methylation. A novel aspect of this work is the generation of a comprehensive genomic atlas of temporal epigenetic and transcriptomic variation, which can serve as an important resource for circadian rhythm studies to investigate diurnal changes in skeletal muscle.

\section{Materials \& methods}

Material and resources used in this article are summarized in Table 1.

\section{C2C12 cell culture}

Mouse C2C12 myoblasts were grown in DMEM with 10\% fetal bovine serum (FBS) and 1\% penstrep. Myoblasts were then seeded into 6-well plates with 50,000 cells per well. At approximately 90\% confluence, cells were differentiated using DMEM with $2 \%$ horse serum and $1 \%$ penicillin-streptomycin. The experiment timeline is outlined in Figure 1A. On day 5 of differentiation, when myotubes were fully maturated, cells were circadian synchronized using 50\% horse serum shock for $2 \mathrm{~h}$ [32], after which media was returned to normal differentiation media. Cells were harvested starting $12 \mathrm{~h}$ after serum shock, every $6 \mathrm{~h}$ for $60 \mathrm{~h}$ in RLT buffer (Qiagen, Hilden, Germany) with $\beta$-mercaptoethanol. RNA and DNA were isolated simultaneously using the RNA/DNA/miRNA AllPrep kit (Qiagen).

\section{RNA sequencing}

RNA was quality assured using the Bioanalyzer instrument (Agilent Technologies, CA, USA) and subjected to the Illumina TruSeq Stranded Total RNA with Ribo-Zero Gold protocol (Illumina, CA, USA) as described. Libraries ( $\mathrm{n}=3$ for each time point) were subjected to 100-bp single-end sequencing on HiSeq 2500 (Illumina) at the Danish National High-Throughput DNA Sequencing Centre. Approximately 22 million reads/sample were assigned to genes with 11,591 genes surviving the expression threshold.

Quantitative reverse transcription polymerase chain reaction (RT-qPCR)

RT-qPCR analysis of Arntl, Nrld1 and $18 S$ (NM_003278) was performed using conventional SYBR green chemistry. Primer sequences $\left(5^{\prime}-3^{\prime}\right)$ are Arntl forward: TAGGATGTGACCGAGGGAAG; reverse: TCAAA- 
(A)

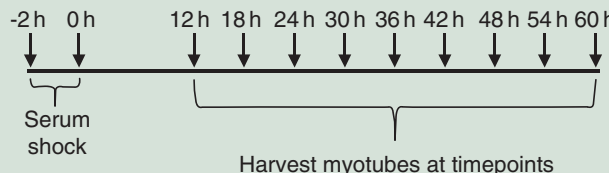

Harvest myotubes at timepoints

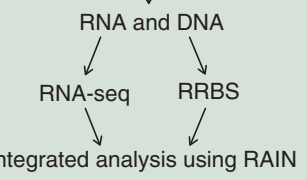

(B)

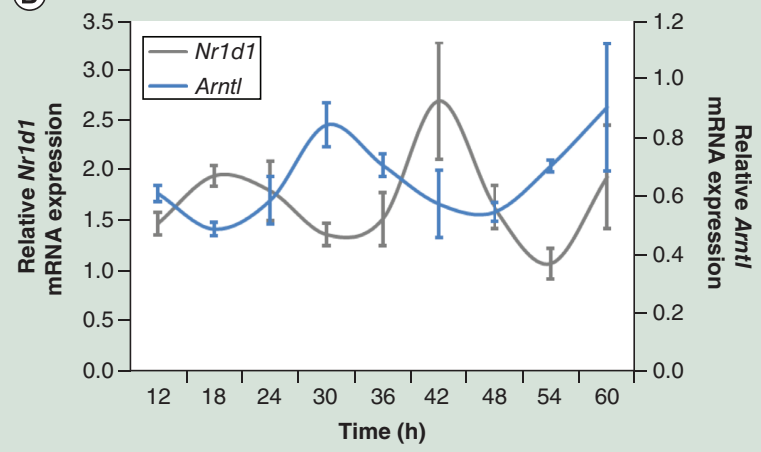

(C)

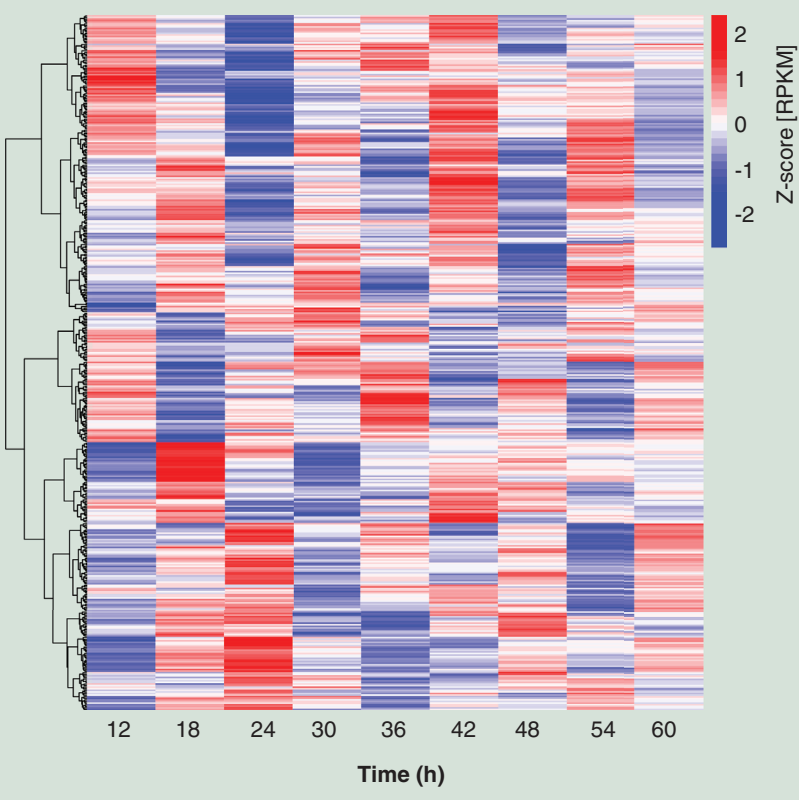

(D)

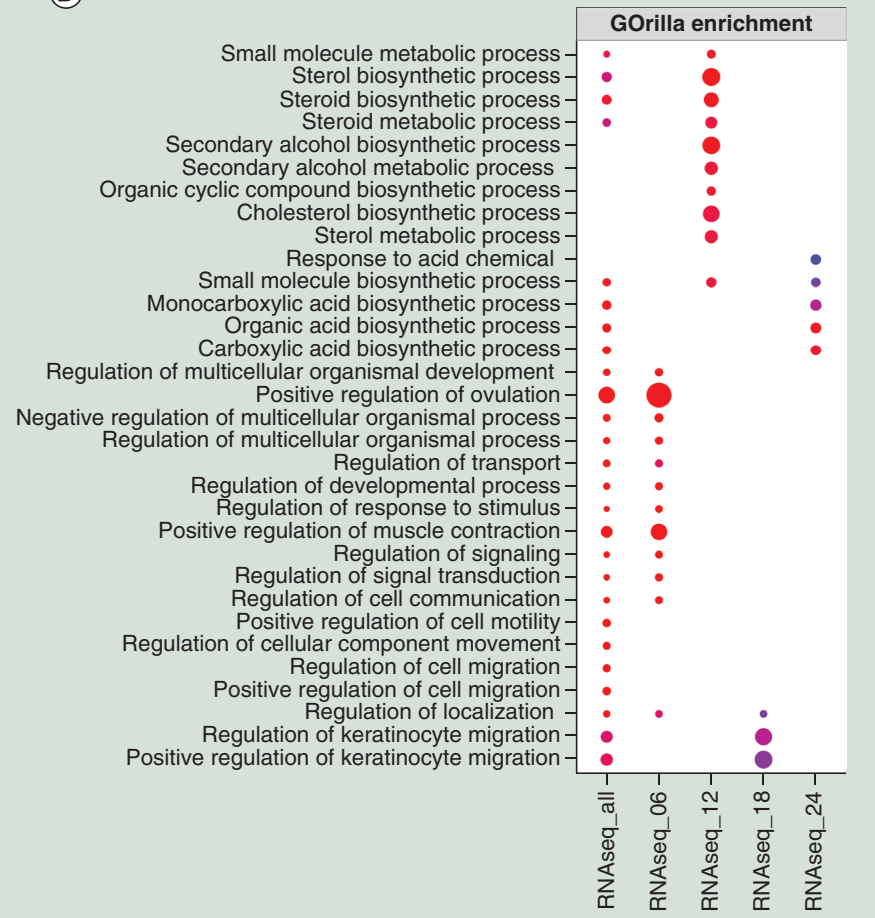

(E)

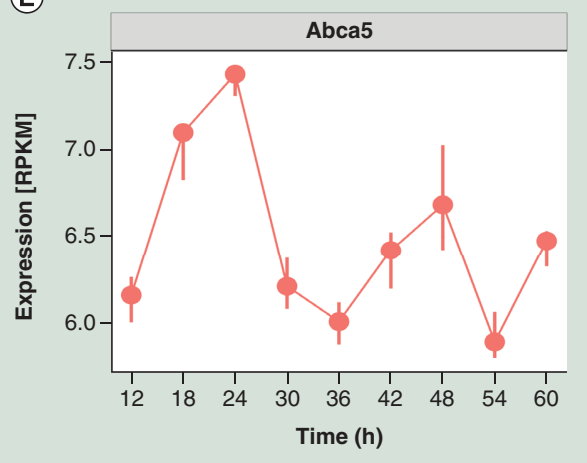

Enrichment
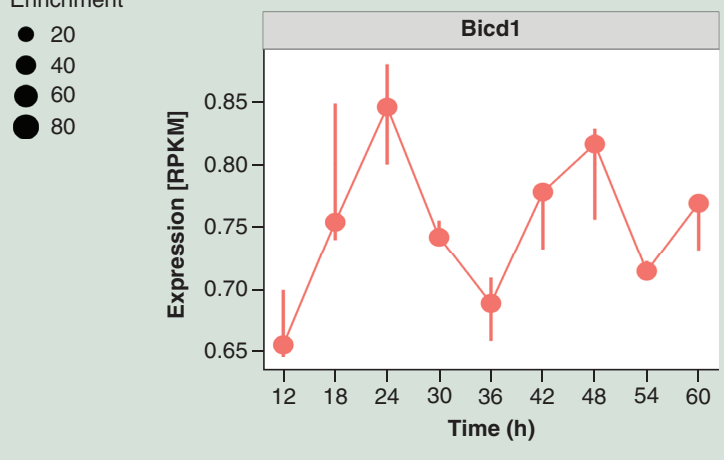

Figure 1. Synchronized muscle cells display robust oscillations of gene expression. (A) C2C12 myotubes were synchronized by serum shock and cells were harvested every $6 \mathrm{~h}$ for $60 \mathrm{~h}$. RNA and DNA was isolated simultaneously and subjected to RNAseq (RNA) and reduced representation bisulfite sequencing (DNA) library preparation, sequencing and bioinformatics analysis for rhythmic behavior. (B) mRNA expression, assessed by RT-qPCR, of the core circadian regulators, Arnt/ and Nr1d1, in synchronized muscle cells. (C) Heatmap of oscillating genes (FDR $\leq 0.1)$ with a $24 \mathrm{~h}$ period as identified by RAIN analysis. Colors indicate time of expression during oscillation (red, high; blue, low). Hierarchical clusters are calculated by 'ward.D2' algorithm using Euclidean distance. A second-degree polynomial was fitted to raw gene expression (Supplementary Figure 1A) normalize the gene expression for trend elimination (Supplementary Figure 1B); (D) Gene ontology analysis of the biological processes of the rhythmic genes for all time points or with a 6-, 12-, 18- or 24 h frequency. (E) mRNA expression of skeletal muscle regulatory genes, Abca5 and Bicd1, as assessed by RNA-seq.

Enrichment: Enrichment score; FDR: False discovery rate; RRBS: Reduced representation bisulfite sequencing; RT-qPCR: Reverse transcription polymerase chain reaction. 


\section{Table 1. Key resources used.}

\begin{tabular}{|c|c|c|c|}
\hline Reagent or resource & Source & Identifier & Ref. \\
\hline \multicolumn{4}{|l|}{ Experimental models: cell lines } \\
\hline - Mouse C2C12 myoblasts & Sigma & 91031101-1VL & \\
\hline \multicolumn{4}{|l|}{ Chemicals } \\
\hline - Horse serum & Sigma & H1270-500ML & \\
\hline - Fetal bovine serum & Sigma & F7524 & \\
\hline - Penstrep & Invitrogen & $15070-063$ & \\
\hline - DMEM & Invitrogen & 41965062 & \\
\hline - B-mercaptoethanol & Biorad & $161-0710$ & \\
\hline - SuperScript ${ }^{\circledR}$ III reverse transcriptase & Invitrogen & 18080044 & \\
\hline - Pfu Turbo Cx hotstart DNA polymerase (500 U) & Agilent & 600412 & \\
\hline - dNTP mix, 100 mM each & NEB & N0446S & \\
\hline- Msp1 & NEB & R0106L & \\
\hline - Klenow fragment $3^{\prime}-5^{\prime}$ & NEB & M0212L & \\
\hline - T4 ligase & NEB & M0202M & \\
\hline \multicolumn{4}{|l|}{ Critical commercial assays } \\
\hline - RNA/DNA/miRNA AllPrep kit & Qiagen & 80224 & \\
\hline - Illumina TruSeq Stranded Total RNA with Ribo-Zero Gold & Illumina & RS-122-2301 & \\
\hline - Agilent RNA 6000 Nano Kit & Agilent & $5067-1511$ & \\
\hline - Agilent High Sensitivity DNA Kit & Agilent & $5067-4626$ & \\
\hline - Qubit ${ }^{\circledR}$ dsDNA HS Assay Kit & Invitrogen & Q32851 & \\
\hline - EZ DNA Methylation Direct ${ }^{\top M}$ kit & Nordic Biosite & D5020 & \\
\hline \multicolumn{4}{|l|}{ Software and algorithms } \\
\hline - RAIN (v1.12.0) & & & [23] \\
\hline - DODR (v0.99.2) & & & [24] \\
\hline - Trim Galore! (v0.3.7) & & & $\mathrm{N} / \mathrm{A}$ \\
\hline - Cutadapt (v1.4.2) & & & [25] \\
\hline - Rsubread (v1.28.1) & & & [26] \\
\hline - featureCounts (v1.6.0) & & & [27] \\
\hline - limma (v3.34.9) & & & [28] \\
\hline - edgeR (v3.24.3) & & & [29] \\
\hline - Bismark (v0.16.3) & & & [30] \\
\hline - GOrilla & & & [31] \\
\hline
\end{tabular}

CAAGCTCTGGCCAAT, $N r 1 d 1$ forward: GTCTCTCCGTTGGCATGTCT; reverse: CCAAGTTCATGGCGCTCT, $18 \mathrm{~s}$ forward AGTCCCTGCCCTTTGTACACA; reverse GATCCGAGGGCCTCACTAAAC.

DNA methylation

Reduced Representation Bisulfite Sequencing (RRBS) was performed as described. DNA samples were incubated with MspI enzyme (NEB, MA, USA) in order to fragment DNA at CCGG positions to enrich for CpG regions. Bisulfite conversion was performed using the EZ DNA methylation Kit (Zymo Research, CA, USA) according to the manufacturer's instructions. DNA was then PCR amplified and ligated to TruSeq (Illumina) sequencing adapters. Libraries ( $\mathrm{n}=3$ for each time point) were subjected to 100-bp single-end sequencing on HiSeq 2500 (Illumina) at the Danish National High-Throughput DNA Sequencing Centre.

\section{Bioinformatic analysis}

RNA-seq reads trimmed by "Trim Galore!" with default settings. Trimmed reads were aligned to mouse genome assembly (mm10) using Rsubread [26]. Aligned reads were summarized into genomic features and gene coverages were counted by featureCounts [27] using the GENCODE annotation [33]. The gene list was filtered to those with a read coverage larger than 0.5 reads per kilobase million, resulting in 11,580 genes. Genes with oscillating behavior with a predefined 24-h period were identified using rhythmicity analysis Involving nonparametric (RAIN) 
algorithm with longitudinal method [23]. RRBS reads were processed with the 'rrbs' setting of Trim Galore v0.3.7 and Cutadapt v1.4.2. Processed reads were mapped to $\mathrm{mm} 10$ followed by derivation of $\mathrm{CpG}$ methylation using Bismark [30]. CpG sites with oscillating methylation were identified using RAIN, with the same settings used for the genes. Differentially rhythmic genes in Miller et al. [33-34] were identified via the DODR [24] algorithm using 'robust' methods with mean centering time series values per gene. The Gene Ontology (GO) enrichment results were obtained from GOrilla [31]. All analyzed genes for RNA-seq and the oscillating CpG sites closest to the transcription start site (TSS) of a gene for RRBS were set as background for each GOrilla analysis.

Heatmaps showing time series and acrophases are generated by 'ward.D2' [35] algorithm using Euclidean distance and geodesic distance, respectively. The matrices used for hierarchical clustering was z-transformed by genes (row) prior to clustering. The gene expression time series heatmap on Figure $1 \mathrm{C}$ was generated by normalized gene expression due to the nonlinear trends in the gene expression:

$$
y_{\text {norm }}=\frac{Y}{\left(a \cdot x+b x^{2}\right)}
$$

while $y_{\text {norm }}$ is the normalized gene expression, $Y$ is the gene expression (reads per kilobase million), $x$ is time, $a$ and $b$ are coefficients for the polynomial fit. Raw data (Supplementary Figure 1) and normalized data (Figure 1C) are shown for some genes on Supplementary Figure 1B.

Acrophases from Miller et al. [34] are aligned to our dataset using $D b p$ (WT) by subtracting the time difference. Time series scatter plots show harmonic regression fit with a linear trend:

$$
y=a+b \cdot \cos \left(\frac{2 \pi}{\tau} x\right)+c \cdot \sin \left(\frac{2 \pi}{\tau} x\right)+d \cdot x
$$

while $y$ is gene expression level, $x$ is time, $\tau$ is period $(24 \mathrm{~h}), a$ is the intercept, $b$ and $c$ are the coefficients for the harmonic regression, $d$ is the coefficient for linear trend.

\section{Results}

Serum shock entrains a functional molecular clock in cultured muscle cells

Following circadian synchronization by serum shock, mouse C2C12 myotubes were collected every $6 \mathrm{~h}$ for a period of $60 \mathrm{~h}$ to cover at least two full circadian cycles (Figure 1A). Prior to RNA sequencing (RNA-Seq), circadian oscillations were confirmed by measuring gene expression of Arntl and $N r 1 d 1$ by real-time RT-qPCR (Figure 1B). Arntl and Nr1d1 mRNA oscillated with a periodicity of approximately $24 \mathrm{~h}$ and in an opposite phase from each other (Figure 1B). After the conformation of circadian oscillation via RT-qPCR, RNA-Seq was performed to create a reference dataset of all oscillating genes in skeletal muscle cells with a period of $24 \mathrm{~h}$ (Supplementary Table 1). The transcriptomic dataset was computationally analyzed using the bioinformatics tool RAIN [23]. We found 439 genes with significant (false discovery rate $[\mathrm{FDR}] \leq 0.1$ ) oscillatory behavior (Figure 1C \& Supplementary Figure 1A \& Supplementary Table 1). The model was further validated by ontology analysis (GOrilla [31]) of genes showing a circadian profile, where 'circadian rhythm' was identified as an enriched biological process in the myotubes (Supplementary Table 2A). GO analysis of genes with a circadian rhythmicity identified terms related to amino acid and lipid transport as dominant biological functions of the circadian genes with many specific terms: carboxylic acid biosynthetic process, organic acid biosynthesis process, monocarboxylic acid biosynthetic process (Figure 1D \& Supplementary Table 2B). Two genes associated with molecule transport showed a particularly distinct circadian oscillatory pattern; Abca5 and Bicdl (Figure 1E).

\section{Synchronized skeletal muscle myotubes partly mimic transcriptional rhythmicity in vivo}

To determine whether synchronized skeletal muscle myotubes in culture mimic circadian oscillations of skeletal muscle in vivo, the RNA-Seq dataset was overlaid with publicly-available microarray data of mouse skeletal muscle sampled every $4 \mathrm{~h}$ for two complete circadian cycles [34]. Cultured mouse skeletal muscle myotubes and wildtype (WT) mouse gastrocnemius skeletal muscle had 46 oscillating genes in common, which represents a specific enrichment (as measured by Fisher's exact test $\mathrm{p}=2.7 \mathrm{e}-04$; Supplementary Table 3A). To identify which of these oscillating genes are controlled by the Bmal1/Clock heterodimer, we compared this analysis with the differentially rhythmic genes from skeletal muscle of Clock mutant mice that shows phase shifts compared with WT mice [34], 
with an activity/rest cycle period that was $4 \mathrm{~h}$ longer [36]. We identified 17 oscillating genes in our RNA-Seq dataset when compared with differentially rhythmic genes from Clock mutant mice microarray data (Figure 2A-B, Supplementary Figure $1 \&$ Supplementary Table 3B), suggesting that the vast majority (29 out of 46) of genes are potential targets of Bmal1/Clock. For example, Per 2 showed a disrupted oscillatory pattern in Clock mutant mice compared with WT mice or compared with our study (Figures 2B \& D). Also, oscillating genes from both datasets are significantly enriched with rhythmic process and circadian rhythm GO terms (Figure 2C). Collectively, our results support the relevance of cultured mouse myotubes as a model for studying circadian rhythms.

\section{CpG DNA methylation is oscillatory on circadian genes}

We next determined whether the rhythmic transcripts identified in the cultured mouse myotubes were associated with oscillating promoter methylation. Using RRBS to assess genome-wide DNA methylation over the 60-h time course, we found DNA methylation occurred almost exclusively in a CG context, with only 1.7 and $1.6 \%$ of methylated cytosines detected at $\mathrm{CHH}$ and CHG sites, respectively (Supplementary Figure 3). RAIN analysis of DNA methylation levels over the $60 \mathrm{~h}$ time course identified oscillatory DNA methylation patterns on 5076 individual CpGs with a phase of $24 \mathrm{~h}$ (FDR $<0.1$; Supplementary Table $4 \mathrm{~A})$ in close proximity ( $98 \%$ of CpGs in $\pm 2 \mathrm{~kb}$ distance to TSS) to 1226 unique genes, referred as CpG-regions (Figure 3A \& Supplementary Table $4 \mathrm{~B})$. Gene ontology analysis of genes close to cycling DNA methylation sites returned broad terms related to organ development processes (Figure $3 \mathrm{~B}$ ). The majority of $\mathrm{CpG}$ regions were in close proximity to TSS, while the distribution of rhythmic CpGs was significantly shifted toward to +500 bp from TSS (Kolmogorov-Smirnov test; $\mathrm{p}<2.2-16$; Figure 3C). RNA-Seq data were overlaid with genome-wide DNA methylation data to determine whether rhythmic DNA methylation is associated with oscillating gene expression. We found 31 out of the 1226 oscillating CpG-regions identified in the RNA-Seq screen had one or more CpG that displayed oscillations in DNA methylation (FDR $<0.1$ ). Within this list, several genes showed marked oscillations in both RNA expression and DNA methylation such as Cry1, Mblac1, Paip2b, PifI and Tspan4 (Figure 4). Moreover, eight out of these 31 genes (Gadd45b, Cry1, Tspan4, Mylk2, Kcnab1, Rhoj, Lancl3 and Prkca) were rhythmic in mouse skeletal muscle (Fisher's exact test, $\mathrm{p}=7.5 \mathrm{e}-04)$. Several CpG sites had an increasing trend in their oscillatory behavior based on their p-value $\left(-\log _{10} F D R\right)$ followed by a decrease, suggesting a potential binding of DNA methyltransferases (DNMTs) and ten-eleven translocation methylcytosine dioxygenases (TETs) in a circadian manner (Figure $4 \&$ Supplementary Figure 5). At the gene expression level, we did not find cyclical regulation of DNMTs or TET enzymes across the time course (Supplementary Figure 4), which does not support regulation of cyclical DNA methylation by intracellular levels of DNMTs or TETs. Collectively, our results indicate that DNA methylation is rhythmic in synchronized muscle cells. However, in cultured myotubes, DNA methylation does not seem to have a major impact on the regulation of gene expression since 2.5\% (31/1226) of rhythmic CpGs are associated with rhythmic gene expression.

\section{Discussion}

Here, we established transcriptomic circadian rhythmicity in cultured skeletal muscle cells and investigated the link between changes in circadian gene expression and DNA methylation at respective gene promoters. We identified novel circadian genes in skeletal muscle and report that a small number of cycling genes are associated with cyclical DNA methylation, notably Cryl, a member of the core clock machinery. We provide a comprehensive transcriptomic and epigenomic resource that reveals pathways controlling circadian regulation in skeletal muscle.

We found that serum shock entrains a functional molecular clock in cultured C2C12 muscle cells, with a subset of genes related to amino acid and lipid transport displaying circadian rhythmicity. We also found genes close to cycling CpG sites were enriched in developmental processes, implying the $\mathrm{C} 2 \mathrm{C} 12$ myotubes are immature. To strengthen the understanding of physiological relevance of our in vitro findings in $\mathrm{C} 2 \mathrm{C} 12$ myotubes, we compared our data with an in vivo study of adult mouse skeletal muscle with a similar design [34]. While C2C12 cells may not fully reflect the physiology of differentiated skeletal muscle, we found that they partly mimicked the transcriptional rhythmicity of adult mouse muscle in vivo [34]. Of note, circadian oscillations in lipid metabolites and transcript profiles of genes encoding for key enzymes involved in their biosynthesis are comparable between cultured primary myotubes and skeletal muscle tissue from the same donor [37], implicating these metabolic and transcriptomic features are preserved between in vitro and in vivo models. Nevertheless, adult skeletal muscle is composed of terminally differentiated cells and, therefore, we cannot exclude the possibility that our results may be specific to $\mathrm{C} 2 \mathrm{C} 12$ myotubes, particularly given the molecular and physiological differences between these models [38]. 
(A)

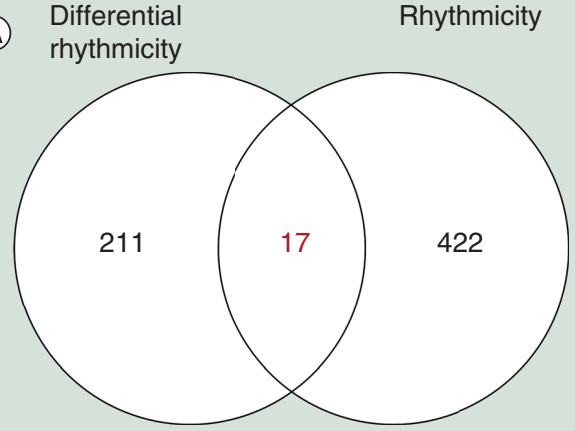

MUT vs WT

(Miller et al., 2007)
(B)

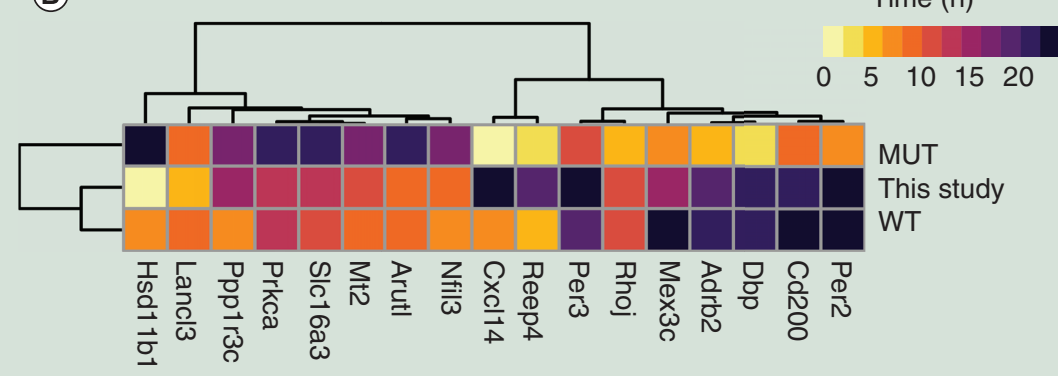

(C)

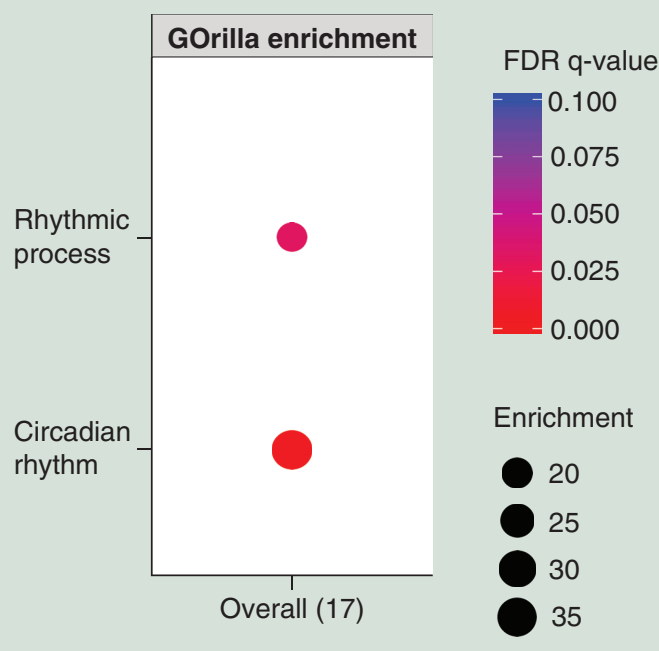

(D)
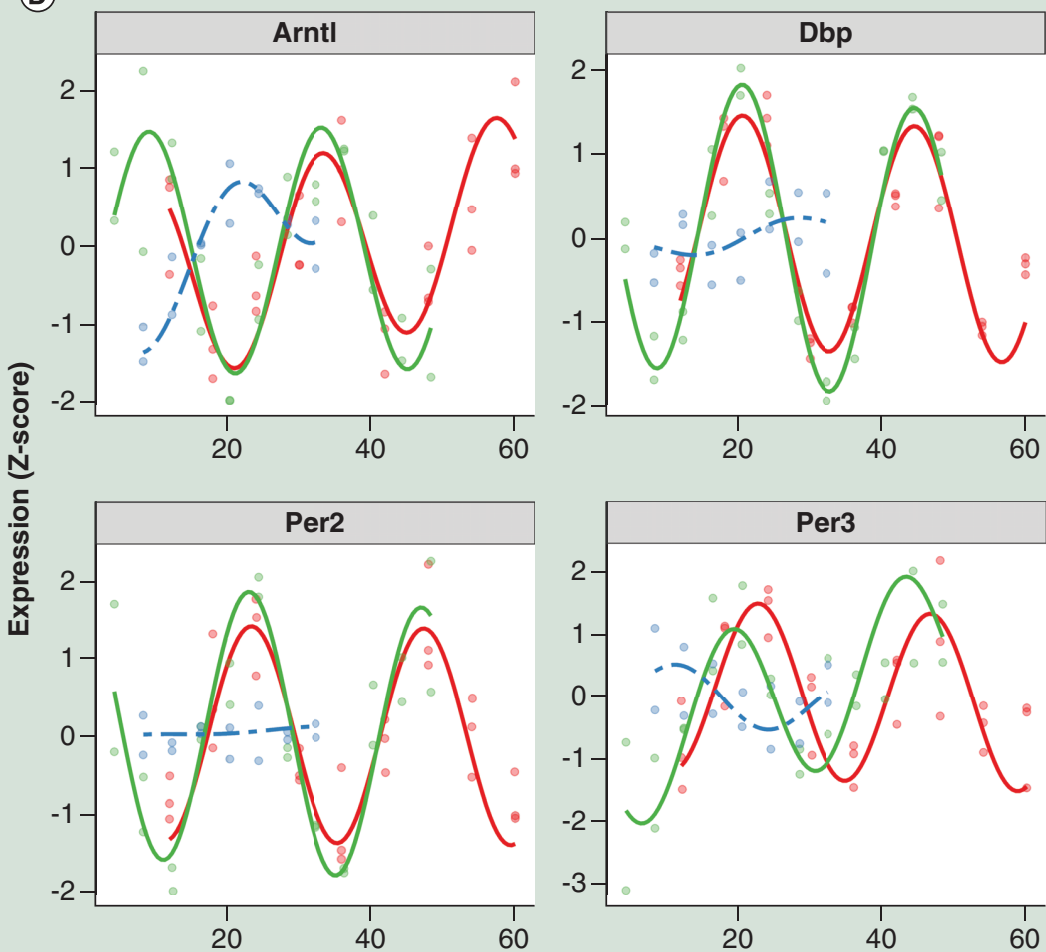

Time (h)

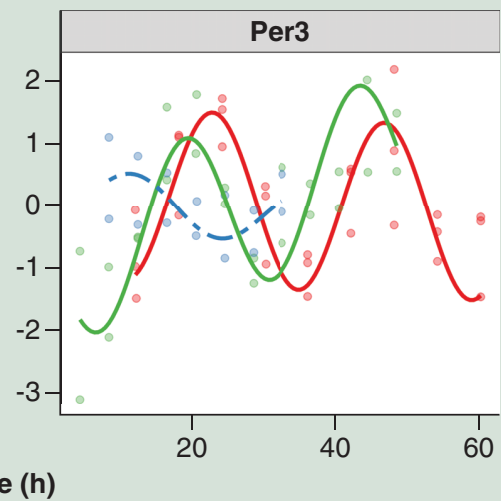

Genotype $\sim$ MUT $\sim$ This study $\sim$ WT

Figure 2. Circadian rhythmicity in C2C12 myotubes recapitulates Clock-regulated genes in mouse skeletal muscle. (A) Venn diagram showing the overlap between differentially rhythmic genes in skeletal muscle from Miller et al. [34] and rhythmic genes identified in cultured myotubes in this study. (B) Heatmap representing circadian phases of rhythmic genes shared between cultured muscle cells ('this study') and mouse skeletal muscle in vivo (WT) and genes with longer period or loss of rhythmicity in skeletal muscle-specific Clock mutant mice (MUT) [34]. Colors indicate time of peak expression (acrophase) during oscillation. Hierarchical clusters are calculated by 'ward.D2' algorithm using geodesic distance. Acrophases for Miller et al. [34] are aligned using Dbp by subtracting the time difference. (C) Gene ontology analysis of the biological processes of the overlapping genes in both datasets. (D) Graphical representation of gene expression oscillations for the Dbp, Per2, Per3 and Arntl in cultured myotubes ('This study') and mouse skeletal muscle in vivo (WT) and genes that lose rhythmicity in skeletal muscle-specific of Clock mutant mice (MUT) [34]. Acrophases are aligned using Dbp by subtracting the time difference, while the Miller et al. [34] time series were shifted $24 \mathrm{~h}$ backwards for better visual overlap. Lines show harmonic regression fit. The rest of the gene expression oscillations are shown in Supplementary Figure 2.

Enrichment: Enrichment score; FDR: False discovery rate; MUT: Clock mutant; WT: Wild-type. 
(A)

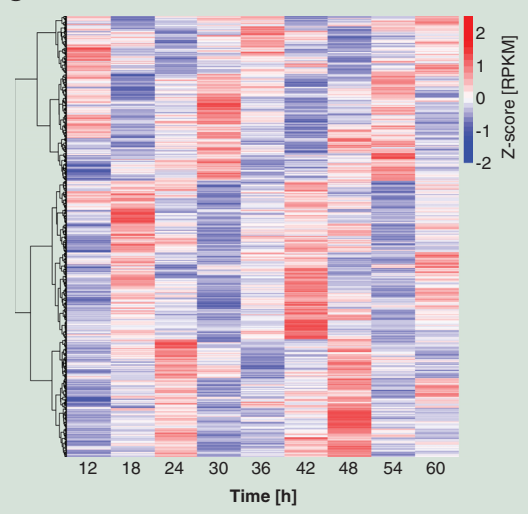

(C)

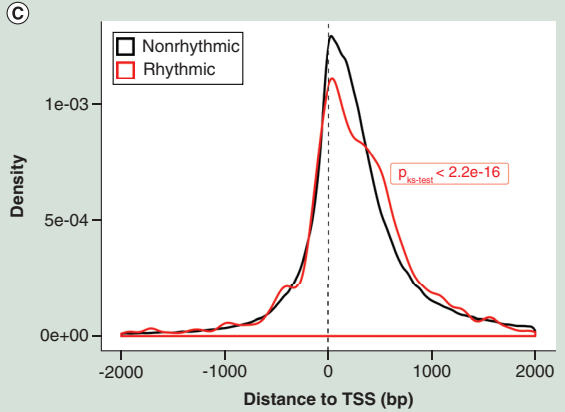

(B)

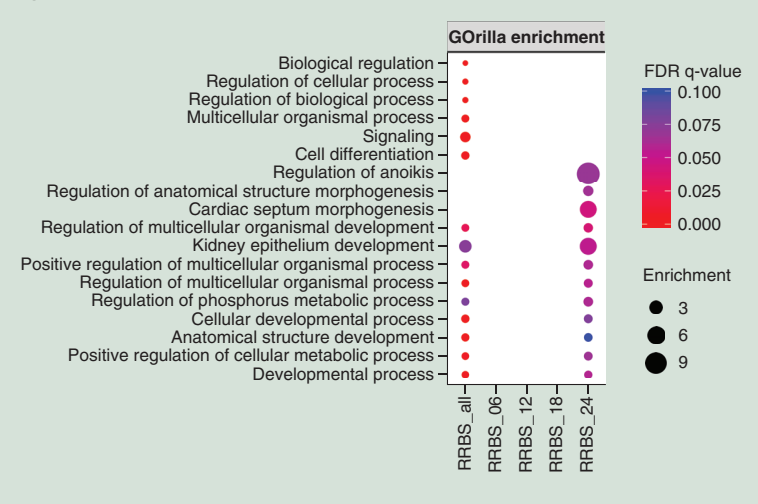

Figure 3. Synchronized C2C12 myotubes display oscillation in promoter DNA methylation. (A) Heatmap of 1226 genes displaying significant oscillation in cytosine methylation. (B) Gene ontology analysis of the biological function and processes of the genes closest to rhythmic CpGs for all time points, or with a 6, 12, 18 or $24 \mathrm{~h}$ frequency. (C) Density distributions of all CpGs (black) and rhythmic CpGs (red) in $\pm 2 \mathrm{~kb}$ range to TSS. $\mathrm{x}$-axis: distance to TSS, $\mathrm{y}$-axis: density. Two distribution are significantly different (Kolmogorov-Smirnov test, $\mathrm{p}$ $<2.2 \mathrm{e}-16)$.

Enrichment: Enrichment score; FDR: False discovery rate; RRBS: Reduced representation bisulfite sequencing; TSS: Transcription start site.

Additional comparative studies between cell culture systems and adult skeletal muscle specimens may further clarify the utility and physiological relevance of cultured myotube models for studying circadian rhythm genomics and metabolism.

One mechanistic question arising from this study is whether interruption of cyclic DNA methylation translates into changes in circadian function and rhythmic gene expression in cultures skeletal myotubes. Although establishing cause-consequence relationships between DNA methylation and gene expression is challenging, new technologies are emerging to highlight the importance of epigenetic modifications on physiological processes, such as targeting DNA methylation modifiers to single cytosines [39,40]. Here we identified methylation changes at single $\mathrm{CpG}$ sites and found a proportion of cycling genes associated with methylation changes on single $\mathrm{CpGs}$ (7.7\%), while cycling gene transcripts shared a smaller proportion (3.7\%). However, the overlap between the cycling genes and nearby CpGs revealed only a few genes $(\mathrm{n}=31)$ suggesting that there is no significant enrichment $(\mathrm{p}=0.54$, Fisher's exact test) between the transcription and the nearby $\mathrm{CpG}$ methylation status. Future studies to manipulate methylation on CpG clusters may address the role of cyclical DNA methylation on the expression of circadian genes. Although gene expression of DNMTs and TETs showed circadian oscillations, together with 5-methylcytosine content in mouse liver [41], none of these genes had $\mathrm{CpG} / \mathrm{mRNA}$ circadian oscillations in mouse skeletal muscle. While tools to address this question are not currently available, approaches to pharmacologically or genetically inhibit the DNMTs or TET enzymes may be useful, with the caveat that this may only reveal indirect relationships. In this regard, a role of the DNA methylation inhibitor 5-azacytidine on the circadian control of gene expression was recently established [42]. Whether inhibition of DNA methylation by 5-azacytidine affects promoter methylation at cycling genes or leads to dysregulation of cycling genes through an indirect epigenetic regulation of transcription factors is unknown. 

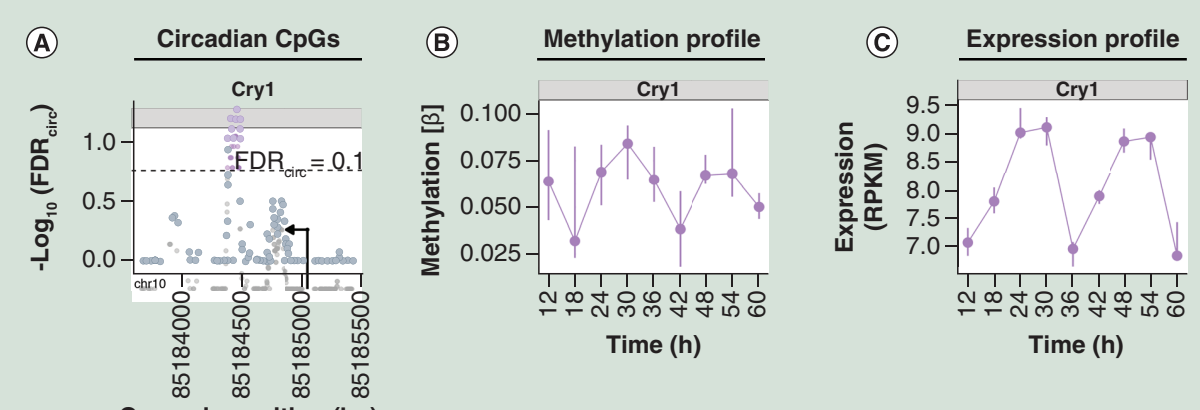

Genomic position (bp)
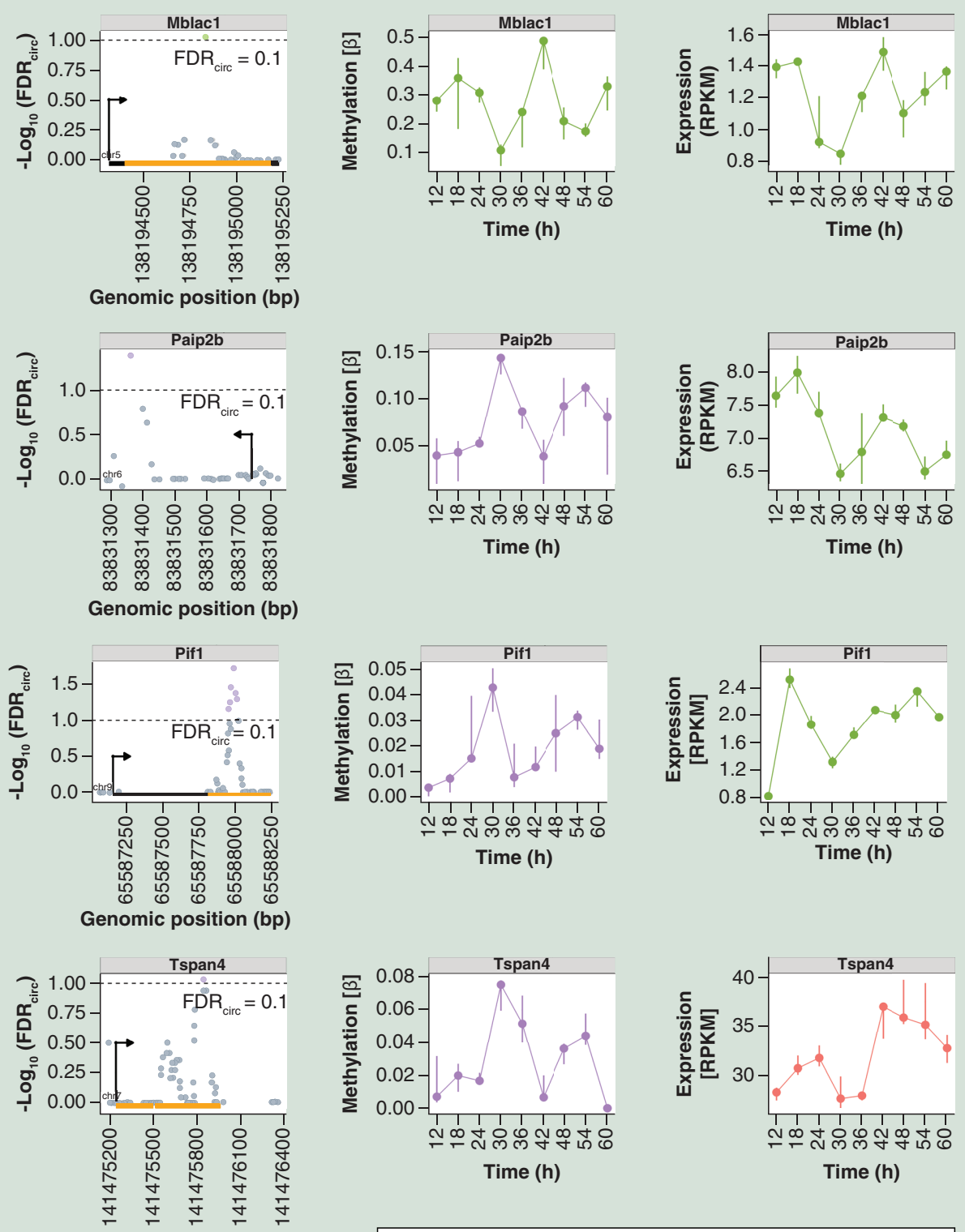

Genomic position (bp)

Nonrhythmic $\cdot 6 \cdot 12 \cdot 18 \cdot 24$ Type $\quad$ CDS $\square$ Exon

Figure 4. The $\mathrm{CpGs}$ and genes both showing circadian rhythmicity. Genomic locations of circadian $\mathrm{CpGs}$, CpG methylation and gene expression profiles over time for Cry1, Mblac1, Paip2b, Pif1 and Tspan4. (A) Circadian CpGs. $x$-axis: Genomic position of CpGs (left bottom, chromosome ID); y-axis, statistical significance of rhythmicity $\left(-\log _{10}\right.$ FDR) calculated by RAIN [23]. (B) CpG methylation profile over time. $x$-axis, time in hours; $y$-axis, methylation $\beta$-values (percent methylation/100). (C) Gene expression profile over time. $x$-axis, time in hours; $y$-axis, gene expression in RPKM. The dots on panel (B) and (C) represent the median while bars show the IQR. Color code represents the peak time (acrophase): purple, 6 h; turquoise, 12 h; green, 18 h; orange, 24 h. Genomic features on first panel are shown as CDS in yellow and exons in black.

CDS: Coding sequence; FDR: False discovery rate; IQR: Interquartile range; RPKM: Reads per kilobase million. 
Questions emerging from this study are whether cycling DNA methylation occurs in skeletal muscle in vivo and whether environmental stimuli such as diet or exercise alter circadian behavior to impact methylation status and subsequent circadian gene expression and phenotypic outcomes. Several lines of evidence indicate a possible association between environmental stimuli and DNA methylation changes on circadian genes. Genome-wide DNA methylation analysis of breast tissue from female shift-workers [43] or blood from male and female shift workers [44] provides evidence for DNA methylation of core clock genes and cancer-related genes, linking altered circadian rhythms with increased risk for specific cancers [43,44]. Time-course analysis of tissues obtained after phase shifts of circadian rhythms could be performed to ascertain the nature of the DNA methylation changes. Such studies may reveal whether the observed changes in DNA methylation are the consequence of a shift in DNA methylation oscillations, a period shift or an overall change throughout all time points. These investigations could offer insight into the role of cyclical DNA methylation changes versus stable DNA methylation changes.

Evidence for fast and cyclical DNA demethylation has emerged from studies showing a rapid cycling of DNA methylation of the pS2/TFF1 gene promoter upon activation by estrogens, with a periodicity of around $100 \mathrm{~min}[45,46]$. In skeletal muscle, contraction induces transient DNA demethylation at promoter regions of exercise-responsive genes [47,48], providing evidence for active dynamic DNA demethylation. While passive DNA demethylation is the result of diluting DNA methylation during cell division by lack of replication of the methylated cytosine on the sister strand, active DNA demethylation requires a series of complex enzymatic processes ending with base-excision repair $[49,50]$. Our results of cyclical DNA methylation changes in nonmitotic cells indicate active demethylation occurs in myocytes.

Although we found many genes with oscillation for both DNA methylation and mRNA expression, the vast majority of cycling methylation at CpG sites were not linked to cyclical gene expression across the time-course. Such lack of association between cyclical DNA methylation and gene expression changes in cultured myotubes over the circadian time course suggests that DNA methylation changes are not sufficient to drive gene expression changes. Similarly, numerous genes with an oscillatory transcription profile were not harboring cyclical DNA methylation at their respective promoters. Thus, other cytosine sites located outside promoters (e.g., in cis-regulatory regions) may control the circadian regulation of gene expression. We propose two putative models for such an event. In the first model, cyclical gene expression is regulated by the cyclical presence of cis-regulatory elements at proximity of the promoter, such as described with the classical model of circadian control by of PER and CRY [18]. In the second model, cyclical DNA methylation regulates the binding of cis-regulatory proteins that are only expressed under a specific environmental or physiological influence, thereby leading to a mechanism of condition-specific cyclical gene expression. Further studies investigating DNA methylation/mRNA expression profiles under specific physiological conditions are warranted to determine the presence of such regulatory models.

Methylation within promoter regions is classically associated with repression of gene expression [46]. Consistently, we found a negative association between DNA methylation and gene expression levels for the majority of genes studied including Paip2b, suggesting that DNA methylation plays an inhibitory function on circadian gene expression. Conversely, for several genes including Cry1 and Mblac1, methylation was positively associated with gene expression. Evidence supports that DNA methylation within gene bodies is positively associated with gene expression [51]. While for Cry 1 and Mblac 1, oscillatory CpG methylation was within distance of the TSS that qualifies for annotation in the promoter region, 26 out of 31 cyclical $\mathrm{CpG}$ sites associated with cyclical gene expression were located downstream from the TSS, which could confer these CpG sites a property to change methylation like other $\mathrm{CpG}$ sites within gene bodies. Since methylation within gene bodies is thought to be secondary to gene expression through interactions between RNA polymerase II and DNMTs [51], cyclical methylation of CpG sites downstream the TSS, when positively associated with gene expression, may be a consequence of, rather than a cause for, cyclical mRNA expression. Further investigations using tools to target DNA methylation at specific cyclical CpG sites, such as dCas9 fused to DNMTs, are required to identify the cytosines involved in the regulation of circadian gene expression. Our study opens novel experimental avenues for manipulating circadian gene expression through the targeted regulation of DNA methylation levels.

\section{Conclusion}

Skeletal muscle has an intrinsic molecular clock, which is associated with metabolic and contractile function. Here, we provide evidence that cultured $\mathrm{C} 2 \mathrm{C} 12$ myotubes maintain a circadian rhythm following serum shock. Moreover, these oscillations are regulated by the mammalian core clock components, ARNTL and NR1D1 and share common oscillating genes with mouse skeletal muscle in vivo and human cells in culture. Circadian oscillation in promoter 
and CpG DNA methylation provides insight into an additional level of gene regulation. Our work provides a comprehensive transcriptomic and epigenomic atlas that may facilitate discovery efforts aimed to uncover new epigenetic pathways controlling circadian regulation in skeletal muscle.

\section{Future perspective}

This study investigates the potential association between the circadian $\mathrm{CpG}$ methylation and the regulation of circadian gene expression in $\mathrm{C} 2 \mathrm{C} 12$ myotubes. Our study reveals that a small fraction of oscillating $\mathrm{CpG}$ methylation is linked to rhythmic gene expression. However, the circadian mechanism behind the changes in $\mathrm{CpG}$ methylation and gene expression is unknown. Whether changes in DNA methylation are the primary driver of these gene expression changes is unresolved. Creating deeper mechanistic insight into this process may lead to the identification of potential biomarkers and development of therapeutic strategies against metabolic diseases. To our knowledge, this is among the first studies to question the potential link between the circadian CpG methylation and the regulation of circadian gene expression in skeletal muscle. Our comprehensive atlas is a resource for future circadian rhythm studies investigating diurnal changes in gene expression and CpG methylation.

\section{Summary points}

- Several rhythmic genes $(n=439)$ and CpG-sites $(n=1126)$ oscillate in circadian-synchronized mouse myotubes.

- Rhythmic and nonrhythmic CpGs are accumulated around transcription start sites, while rhythmic CpGs are shifted toward +500 bp from transcription start sites.

- Gene ontology terms for circadian genes are enriched for skeletal muscle metabolism.

- Circadian CpG methylation is found near genes regulating developmental processes.

- Gene expressions of enzymes regulating DNA methylation and TETs are not rhythmic.

- A small fraction of oscillating CpG methylation sites are associated with rhythmic expression of genes $(n=31)$.

- The circadian gene Cry1 is subject to marked rhythmicity of CpG methylation.

\section{Supplementary data}

To view the supplementary data that accompany this paper please visit the journal website at: www.futuremedicine.com/doi/sup $\mathrm{pl} / 10.2217 /$ epi-2019-0391

\section{Author contributions}

A Altıntaş performed bioinformatics analysis and figure generation and wrote the manuscript; RC Laker performed the experiments, analyzed the data and wrote the manuscript; C Gard performed bioinformatics analysis and figure generation; R Barrès designed the experiments, provided expert advice and wrote the manuscript; JR Zierath designed the experiments, provided expert advice and wrote the manuscript.

\section{Acknowledgments}

The authors would like to thank Z Gerhart-Hines for his expert advice.

\section{Financial \& competing interests disclosure}

The Novo Nordisk Foundation Centre for Basic Metabolic Research is an independent research center at the University of Copenhagen partially funded by an unrestricted donation from the Novo Nordisk Foundation (NNF18CC003490). This work was funded by a Novo Nordisk Foundation Challenge Grant (NNF14OC0011493) and the Swedish Research Council (2015-00165). The authors have no other relevant affiliations or financial involvement with any organization or entity with a financial interest in or financial conflict with the subject matter or materials discussed in the manuscript apart from those disclosed.

No writing assistance was utilized in the production of this manuscript.

Data availability

Sequencing data are archived (GSE131844) for public access at the Gene Expression Omnibus (http://www.ncbi.nlm.nih.gov/geo).

\section{Open access}

This work is licensed under the Attribution-NonCommercial-NoDerivatives 4.0 Unported License. To view a copy of this license, visit http://creativecommons.org/licenses/by-nc-nd/4.0/ 


\section{References}

1. Hastings MH, Reddy AB, Maywood ES. A clockwork web: circadian timing in brain and periphery, in health and disease. Nat. Rev. Neurosci. 4(8), 649-661 (2003).

2. Stratmann M, Schibler U. Properties, entrainment and physiological functions of mammalian peripheral oscillators. J. Biol. Rhythms 21(6), 494-506 (2006).

3. Antunes LC, Levandovski R, Dantas G, Caumo W, Hidalgo MP. Obesity and shift work: chronobiological aspects. Nutr. Res. Rev. 23(1), 155-168 (2010).

4. Esquirol Y, Bongard V, Mabile L, Jonnier B, Soulat JM, Perret B. Shift work and metabolic syndrome: respective impacts of job strain, physical activity and dietary rhythms. Chronobiol. Int. 26(3), 544-559 (2009).

5. Pan A, Schernhammer ES, Sun Q, Hu FB. Rotating night shift work and risk of type 2 diabetes: two prospective cohort studies in women. PLoS Med. 8(12), e1001141 (2011).

6. Ko CH, Takahashi JS. Molecular components of the mammalian circadian clock. Hum. Mol. Genet. 15(Spec. No. 2), R271-R277 (2006).

7. Defronzo RA, Sherwin RS, Kraemer N. Effect of physical training on insulin action in obesity. Diabetes 36(12), 1379-1385 (1987).

8. Dyar KA, Ciciliot S, Tagliazucchi GM et al. The calcineurin-NFAT pathway controls activity-dependent circadian gene expression in slow skeletal muscle. Mol. Metab. 4(11), 823-833 (2015).

9. Hansen J, Timmers S, Moonen-Kornips E et al. Synchronized human skeletal myotubes of lean, obese and type 2 diabetic patients maintain circadian oscillation of clock genes. Sci. Rep. 6, 35047 (2016).

10. Hodge BA, Wen Y, Riley LA et al. The endogenous molecular clock orchestrates the temporal separation of substrate metabolism in skeletal muscle. Skelet. Muscle 5, 17 (2015).

11. McCarthy JJ, Andrews JL, McDearmon EL et al. Identification of the circadian transcriptome in adult mouse skeletal muscle. Physiol. Genomics 31(1), 86-95 (2007).

12. Andrews JL, Zhang X, Mccarthy JJ et al. CLOCK and BMAL1 regulate MyoD and are necessary for maintenance of skeletal muscle phenotype and function. Proc. Natl Acad. Sci. USA 107(44), 19090-19095 (2010).

13. Van Moorsel D, Hansen J, Havekes B et al. Demonstration of a day-night rhythm in human skeletal muscle oxidative capacity. Mol. Metab. 5(8), 635-645 (2016).

14. Peek CB, Levine DC, Cedernaes J et al. Circadian clock interaction with HIF1alpha mediates oxygenic metabolism and anaerobic glycolysis in skeletal muscle. Cell Metab. 25(1), 86-92 (2017).

15. Perrin L, Loizides-Mangold U, Skarupelova $S$ et al. Human skeletal myotubes display a cell-autonomous circadian clock implicated in basal myokine secretion. Mol. Metab. 4(11), 834-845 (2015).

16. Harfmann BD, Schroder EA, Kachman MT, Hodge BA, Zhang X, Esser KA. Muscle-specific loss of Bmal1 leads to disrupted tissue glucose metabolism and systemic glucose homeostasis. Skelet. Muscle 6, 12 (2016).

17. Aguilar-Arnal L, Katada S, Orozco-Solis R, Sassone-Corsi P. NAD(+)-SIRT1 control of H3K4 trimethylation through circadian deacetylation of MLL1. Nat. Struct. Mol. Biol. 22(4), 312-318 (2015).

18. Aguilar-Arnal L, Sassone-Corsi P. The circadian epigenome: how metabolism talks to chromatin remodeling. Curr. Opin. Cell Biol. 25(2), 170-176 (2013).

19. Koike N, Yoo SH, Huang HC et al. Transcriptional architecture and chromatin landscape of the core circadian clock in mammals. Science 338(6105), 349-354 (2012).

20. Masri S, Sassone-Corsi P. The circadian clock: a framework linking metabolism, epigenetics and neuronal function. Nat. Rev. Neurosci. 14(1), 69-75 (2013).

21. Vollmers C, Schmitz RJ, Nathanson J, Yeo G, Ecker JR, Panda S. Circadian oscillations of protein-coding and regulatory RNAs in a highly dynamic mammalian liver epigenome. Cell Metab. 16(6), 833-845 (2012).

22. Azzi A, Dallmann R, Casserly A et al. Circadian behavior is light-reprogrammed by plastic DNA methylation. Nat. Neurosci. 17(3), 377-382 (2014).

23. Thaben PF, Westermark PO. Detecting rhythms in time series with RAIN. J. Biol. Rhythms 29(6), 391-400 (2014).

24. Thaben PF, Westermark PO. Differential rhythmicity: detecting altered rhythmicity in biological data. Bioinformatics 32(18), 2800-2808 (2016).

25. Martin M. Cutadapt removes adapter sequences from high-throughput sequencing reads. EMBnet.journal 17(1), 10 (2011).

26. Liao Y, Smyth GK, Shi W. The Subread aligner: fast, accurate and scalable read mapping by seed-and-vote. Nucleic Acids Res. 41(10), e108 (2013).

27. Liao Y, Smyth GK, Shi W. FeatureCounts: an efficient general purpose program for assigning sequence reads to genomic features. Bioinformatics 30(7), 923-930 (2014).

28. Ritchie ME, Phipson B, Wu D et al. Limma powers differential expression analyses for RNA-sequencing and microarray studies. Nucleic Acids Res. 43(7), e47 (2015). 
29. Robinson MD, Mccarthy DJ, Smyth GK. edgeR: a bioconductor package for differential expression analysis of digital gene expression data. Bioinformatics 26(1), 139-140 (2010).

30. Krueger F andrews SR. Bismark: a flexible aligner and methylation caller for Bisulfite-Seq applications. Bioinformatics 27(11), 1571-1572 (2011).

31. Eden E, Navon R, Steinfeld I, Lipson D, Yakhini Z. GOrilla: a tool for discovery and visualization of enriched GO terms in ranked gene lists. BMC Bioinformatics 10, 48 (2009).

32. Balsalobre A, Damiola F, Schibler U. A serum shock induces circadian gene expression in mammalian tissue culture cells. Cell. 93(6), 929-37 (1998).

33. Frankish A, Diekhans M, Ferreira AM et al. GENCODE reference annotation for the human and mouse genomes. Nucleic Acids Res. 47(D1), D766-D773 (2019).

34. Miller BH, Mcdearmon EL, Panda $S$ et al. Circadian and CLOCK-controlled regulation of the mouse transcriptome and cell proliferation. Proc. Natl Acad. Sci. USA 104(9), 3342-3347 (2007).

35. Murtagh F, Legendre P. Ward's hierarchical agglomerative clustering method: which algorithms implement Ward's criterion? J. Classif. 31(3), 274-295 (2014).

36. Vitaterna MH, King DP, Chang AM et al. Mutagenesis and mapping of a mouse gene, Clock, essential for circadian behavior. Science 264(5159), 719-725 (1994).

37. Loizides-Mangold U, Perrin L, Vandereycken B et al. Lipidomics reveals diurnal lipid oscillations in human skeletal muscle persisting in cellular myotubes cultured in vitro. Proc. Natl Acad. Sci. USA 114(41), E8565-E8574 (2017).

38. Abdelmoez AM, Sardon Puig L, Smith JA et al. Comparative profiling of skeletal muscle models reveals heterogeneity of transcriptome and metabolism. Am. J. Physiol. Cell Physiol. doi:10.1152/ajpcell.00540.2019 (2019) (Epub ahead of print).

39. Lei Y, Zhang X, Su J et al. Targeted DNA methylation in vivo using an engineered dCas9-MQ1 fusion protein. Nat. Commun. 8, 16026 (2017).

40. Amabile A, Migliara A, Capasso P et al. Inheritable silencing of endogenous genes by hit-and-run targeted epigenetic editing. Cell 167(1), 219-232 (2016).

41. Xia L, Ma S, Zhang Y et al. Daily variation in global and local DNA methylation in mouse livers. PLoS ONE 10(2), e0118101 (2015).

42. Tomita T, Kurita R, Onishi Y. Epigenetic regulation of the circadian clock: role of 5-aza-2'-deoxycytidine. Biosci. Rep. 37(3), 1-10 (2017).

43. Zhu Y, Stevens RG, Hoffman AE et al. Epigenetic impact of long-term shiftwork: pilot evidence from circadian genes and whole-genome methylation analysis. Chronobiol. Int. 28(10), 852-861 (2011).

44. Bhatti P, Zhang Y, Song X et al. Nightshift work and genome-wide DNA methylation. Chronobiol. Int. 32(1), 103-112 (2015).

45. Kangaspeska S, Stride B, Metivier R et al. Transient cyclical methylation of promoter DNA. Nature 452(7183), 112-115 (2008).

46. Metivier R, Gallais R, Tiffoche C et al. Cyclical DNA methylation of a transcriptionally active promoter. Nature 452(7183), 45-50 (2008).

47. Barres R, Yan J, Egan B et al. Acute exercise remodels promoter methylation in human skeletal muscle. Cell Metab. 15(3), 405-411 (2012).

48. Pattamaprapanont P, Garde C, Fabre O, Barres R. Muscle contraction induces acute hydroxymethylation of the exercise-responsive gene Nr4a3. Front. Endocrinol. (Lausanne) 7, 165 (2016).

49. Guo JU, Su Y, Zhong C, Ming GL, Song H. Hydroxylation of 5-methylcytosine by TET1 promotes active DNA demethylation in the adult brain. Cell 145(3), 423-434 (2011).

50. Wu SC, Zhang Y. Active DNA demethylation: many roads lead to Rome. Nat. Rev. Mol. Cell Biol. 11(9), 607-620 (2010).

51. Yang X, Han H, De Carvalho DD, Lay FD, Jones PA, Liang G. Gene body methylation can alter gene expression and is a therapeutic target in cancer. Cancer Cell 26(4), 577-590 (2014). 
